COMPETING INTERESTS STATEMENT

The authors declare competing financial interests: details accompany the full-text HTML version of the paper at http://www.nature.com/naturemedicine/.

1. Rothenberg, M.E..J. Allergy Clin. Immunol. 113 11-28; quiz 29 (2004).

2. Rothenberg, M.E. \& Hogan, S.P. Annu. Rev. Immunol. 24, 147-174 (2006).
3. Yousefi, S. et al. Nat. Med. 949-953 (2008).

4. Jacobsen, E.A., Taranova, A.G., Lee, N.A. \& Lee, J.J. J. Allergy Clin. Immunol. 119, 1313-1320 (2007).

5. Yang, D. et al. J. Exp. Med. 205, 79-90 (2008).

6. Jacobsen, E.A. et al. J. Exp. Med. 205, 699-710 (2008).

7. Walsh, E.R. et al. J. Exp. Med. 205, 1285-1292 (2008).

8. Mishra, A., Hogan, S.P., Lee, J.J., Foster, P.S. \&
Rothenberg, M.E. J. Clin. Invest. 103, 1719-1727 (1999).

9. Brinkmann, V. et al. Science 303, 1532-1535 (2004).

10. Buchanan, J.T. et al. Curr. Biol. 16, 396-400 (2006).

11. Fuchs, T.A. et al. J. Cell Biol. 176, 231-241 (2007)

12. Gray, M.W., Burger, G. \& Lang, B.F. Science $\mathbf{2 8 3}$ 1476-1481 (1999).

\title{
Disarming the malaria parasite
}

\author{
Christian R Engwerda \& Michael F Good
}

\section{Investigation of a genetically attenuated malaria parasite—which infects but does not kill its host—provides insight into how to develop a malaria vaccine (pages 954-958).}

Malaria kills more than 1 million people each year and is the leading cause of death in children in the developing world; each year, there are around 500 million clinical episodes of this disease ${ }^{1,2}$. There is no effective vaccine despite many years of effort, and drug treatment is continually undermined by the development of drug-resistant parasite strains and insecticide resistance of the vector. New control strategies are desperately needed.

A safe and effective vaccine remains a preferred option because of the potential for low-cost production and administration, widespread delivery and impact on the disease. As reported in this issue of Nature Medicine, Ting et al. ${ }^{3}$ have created a genetically attenuated malaria parasite capable of inducing potent, protective host immunity against malaria blood-stage infection. They have generated a valuable tool to understand how to make an effective malaria vaccine and design better ways to treat malaria with drugs $^{3}$.

The development of a suitable vaccine is made difficult by the relatively complex life cycle of malaria parasites (Fig. 1). Nevertheless, three broad vaccine approaches are actively being investigated, each targeting different stages of the parasite life cycle: pre-erythrocytic or liver stage, erythrocytic or blood stage and sexual stage.

The first two approaches aim to protect individuals, whereas the last approach is intended to induce an immunity that would block transmission to others without directly

Christian R. Engwerda and Michael F. Good are at the Australian Centre for Vaccine Development, Queensland Institute of Medical Research, 300 Herston Road, Herston QLD 4006, Australia. e-mail: Michael.Good@qimr.edu.au or chrisE@qimr.edu.au protecting the vaccinated individual ${ }^{4}$. Each approach has merit, and there are strong arguments to combine different approaches into a single vaccine for additive protection.

In Plasmodium, purines are required for RNA and DNA synthesis but cannot be synthesized by the parasite de novo ${ }^{5}$. Hence, Plasmodium parasites have evolved a unique but simple purine pathway in which purine nucleoside phosphorylase (PNP) promotes both purine salvage and recycling ${ }^{6}$. Ting et al. ${ }^{3}$ evaluated the importance of PNP in the lethal rodent parasite Plasmodium yoelii YM by generating PNP-deficient parasites ( $\triangle P y P N P$ clones).

These genetically modified parasites had no apparent defects in development in blood cells, but their growth was impaired relative to wild-type parasites. Furthermore, although the sexual stages (gametocytes) could develop and be transmitted to mosquitoes from the blood, progression of the life cycle in the mosquito was blocked, indicating a crucial role for PNP in the sexual stage of the parasite life cycle. Blood stage infections with these genetically attenuated parasites did not kill mice (even though the originating wild-type parasite was lethal), and the mice controlled parasite growth. Importantly, mice that cleared $\triangle P y P N P$ strain infections were immune to subsequent challenge with wild-type P. yoelii YM. These immune mice were also protected against a different, albeit nonlethal, parasite strain $(P$. yoelii 17XNL), delivered as either a blood stage challenge or via a sporozoite inoculation by mosquito bite. This strain is the first defined example of a genetically attenuated malaria blood stage parasite capable of inducing immunity.

One potential application for these genetically attenuated parasites is in a whole- parasite vaccine. There is a resurgence of interest in whole-parasite vaccines ${ }^{7}$, owing to the lack of success in developing malaria subunit vaccines that comprise one or a few parasite antigens, along with issues regarding the ability of malaria parasites to mutate or switch gene expression to render host immunity to new parasite challenge ineffective.

Irradiated sporozoites are potent vaccines capable of inducing effective parasite-specific cytolytic $\left(\mathrm{CD}^{+}\right) \mathrm{T}$ cell responses that control liver stage infection ${ }^{8,9}$ and, hence, subsequent blood stage infection (Fig. 1). The development of a genetically attenuated (with one or two genes knocked out) Plasmodium parasite unable to develop fully in the liver but capable of inducing strong immunity against a subsequent challenge by wild-type sporozoites in a mouse model suggested that genetically attenuated Plasmodium sporozoites could be used as a vaccine for humans ${ }^{8}$.

One concern with using either genetically attenuated or even radiation-attenuated sporozoites as vaccines is that any reversion to virulence (in the case of genetically attenuated parasites) or inadequate irradiation (in the case of irradiated sporozoites) could result in the establishment of blood stage infection (Fig. 1). An intriguing possibility would be to develop PNP-deficient sporozoites able to pass through the liver stage to induce immunity against pre-erythrocytic and blood stage parasites. However, this approach is currently not feasible, because PNP-deficient parasites fail to develop fully in the mosquito, and transmission from mosquito to host is required for sporozoite development.

Although the experiments with $\triangle P y P N P$ parasites are of particular interest in that the parasites have been attenuated by a defined genetic mutation, the principle of using 


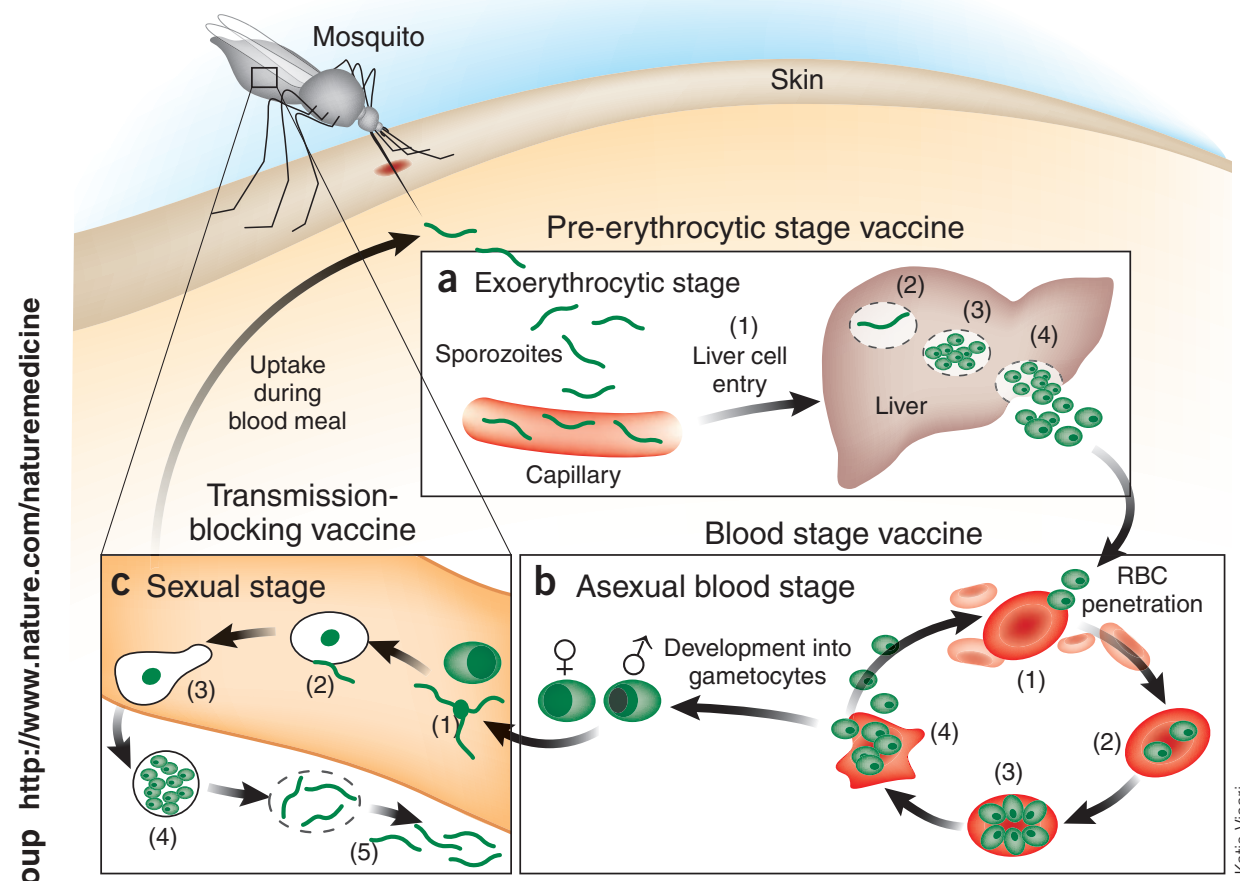

Figure 1 The life cycle of the Plasmodium parasite has three distinct stages. Malaria sporozoites are introduced into humans when an infected female Anopheles mosquito takes a blood meal. (a) During the exoerythrocytic stage, sporozoites make their way through the blood to the liver via Kupffer cells (1). Sporozoites invade numerous hepatocytes (2) and develop into the exoerythrocytic form (EEF), also known as tissue schizonts (3). These EEFs contain many merozoites, which are released and circulate in the blood upon EEF rupture (4). (b) The asexual blood stage commences when the merozoite invades a red blood cell (RBC; 1 ). Within the RBC, the merozoite develops into a trophozoite (2) and then into a schizont (3). The asexual blood stage cyclically continues as schizonts rupture, releasing merozoites for further invasion of RBCs (4 and 1). Male and female gametocytes are also produced during this stage and are taken up by the mosquito when feeding (see panel c). (c) The sexual stage takes place within the gut of the (2) mosquito. Inside the gut, the gametocytes (1) are released and fuse to form a zygote (2), which in turn develops into an ookinete (3). The ookinete crosses the midgut epithelial cell wall. Between the epithelial cell wall and the basal lamina of the midgut, the ookinete develops into an oocyst (4). The oocyst produces thousands of sporozoites, which are released into the hemocele (the body cavity) and are carried in the hemolymph to the salivary glands (5), ready for injection into the next host. The experiments of Ting et al. ${ }^{3}$ provide insight into development of a blood stage vaccine and provide a tool to better understand the development of immunity to blood stage infection.

blood stage parasites that are genetically attenuated by irradiation and that induce blood stage immunity is not new ${ }^{10}$. There are probably many genes that could be targeted to develop a genetically defined attenuated parasite. Furthermore, some of these attenuated parasites may be able to produce sporozoites that develop in the liver but have impaired blood stage growth, and so could be tested as a combined blood and liver stage vaccine to provide first- and second-line vaccine-induced immune defense.

Whole-parasite vaccine approaches face many obstacles, including the need to address serious safety concerns regarding the ability of attenuated parasites to induce disease in some individuals or undergo genetic reversion. Indeed, a major concern with using an attenuated parasite similar to $P$. yoelii $\triangle P y P N P$ is the level of parasite burden reached before infection resolves. In some individuals, even modest blood parasitemia may result in severe malaria complications. Other obstacles facing whole-parasite vaccines include potential concerns about red blood cell proteins contaminating the vaccine and the need for refrigeration of the vaccine in remote areas.

Another promising approach to reduce the burden of malaria is intermittent preventative treatment $(\mathrm{IPT})^{11}$. A therapeutic course of an antiparasite drug is administered for a prescribed period, regardless of infection, with the intention of clearing existing infections and protecting against new infection until the drug levels decline. IPT is effective at protecting pregnant women from placental malaria and their babies from malariaassociated low birth weight ${ }^{12}$. More recently, a review of published trials revealed that IPT reduced the incidence of clinical malaria in infants by $20-30 \%^{13}$. The effectiveness of IPT is likely to involve the ability of drugs to kill parasites while allowing the development of host immunity—because drug treatment and immune mechanisms can act synergistically to clear infections ${ }^{14}$.

At present, our understanding of the effects of IPT on host immune responses is limited, although it is an area of active investigation. The attenuated blood stage parasites offer an opportunity to define the role of host immune responses in infection-cure regimens such as IPT in a defined experimental setting, given the limited blood stage growth of $P$. yoelii $\triangle P y P N P$ and of malaria parasites in hosts receiving IPT (most IPT drugs act against blood stages and allow lowdose blood stage parasitemia). These types of studies could be conducted in experimental models of blood stage challenge or in human trials. The information gained from such studies would be valuable in identifying the appropriate immune responses to target via vaccination.

The development of malaria research tools, such as those described by Ting et al. ${ }^{3}$, provide new avenues of study for the malaria research community. These tools can either be directly developed as vaccines or be used to better understand the immune responses necessary to prevent the development of severe malaria disease.

1. Snow, R.W., Craig, M.H., Newton, C.R. \& Steketee, R.W. http://www.cdc.gov/malaria/pdf/snow_wp11.pdf (2003).

2. Snow, R.W., Guerra, C.A., Noor, A.M., Myint, H.Y. \& Hay, S.I. Nature 434, 214-217 (2005).

3. Ting, L.-M. et al. Nat. Med. 14, 954-958 (2008)

4. Dinglasan, R.R. \& Jacobs-Lorena, M. Trends Parasitol. 24, 364-370 (2008).

5. Ting, L.M. et al. J. Biol. Chem. 280, 9547-9554 (2005).

6. Wykes, M. \& Good, M.F. Int. J. Parasitol. 37, 705-712 (2007).

7. Weiss, W.R., Sedegah, M., Beaudoin, R.L., Miller, L.H \& Good, M.F. Proc. Natl. Acad. Sci. USA 85, 573-576 (1988).

8. Luke, T.C. \& Hoffman, S.L. J. Exp. Biol. 206, 38033808 (2003).

9. Mueller, A.K., Labaied, M., Kappe, S.H. \& Matuschewski, K. Nature 433, 164-167 (2005).

10. Waki, S., Yonome, I. \& Suzuki, M. Exp. Parasitol. 62 316-321 (1986).

11. O'Meara, W.P., Smith, D.L. \& McKenzie, F.E. PLoS Med. 3, e141 (2006).

12. Shulman, C.E. et al. Lancet 353, 632-636 (1999).

13. Committee on the perspectives on the role of intermittent preventive treatment for malaria in infants. Assessment of the Role of Intermittent Preventive Treatment for Malaria in Infants: Letter Report. 1-64 (The National Academies Press, Washington, DC 2008).

14. Djimde, A.A. et al. Am. J. Trop. Med. Hyg. 69, 558-563 (2003). 